SECTION 4. Computer science, computer engineering and automation.

Nick Voloshyn

Graduate student, Department of Hybrid Modeling and Controlling Systems in Energy Engineering, Pukhov Institute for Modeling in Energy Engineering, NAS of Ukraine,

Ukraine

Nick.Voloshyn@yandex.ua

\title{
EFFICIENT AND ROBUST RECOGNITION OF THE OBJECTS ON THE GREY- VALUED PICTURES
}

\begin{abstract}
The results of investigations of a problem of object detection on the image are described in the article. Methods for estimation of dissimilarity between pictures and methods of the pictures matching are investigated as well as procedures of picture preprocessing are proposed in this article.
\end{abstract}

Key words: recognition, segmentation, noise reducing.

\section{УДК 004.942, 004.451.24, 57.087}

\section{ЭФФЕКТИВНОЕ И НАДЕЖНОЕ РАСПОЗНАВАНИЕ ОБЪЕКТОВ НА ПОЛУТОНОВЫХ ИЗОБРАЖЕНИЯХ}

\begin{abstract}
Аннотация: В данной статье описань результатьл исследований задачи обнаружения объекта наблюдения на изображении. Также в статье сформированы методы оченки несходства между фотографиями и исследуются методы совпадения фотографий, а также проиедуры предварительной обработки изображения.
\end{abstract}

Ключевые слова: распознавание, сегментащия, подавление шумов

Изображение объектов при различном освещении могут радикально различаться. Это порождает значительные трудности в прикладных задачах анализа и интерпретации изображения реальных сцен с несколькими объектами, в которых решение не должно зависеть от условий регистрации изображения [1]. Например, выделения неизвестного объекта на фоне известной местности, распознавание, классификация образов по их изображению, задачи совмещения изображения одной и той же сцены, полученных в различных спектральных диапазонах и т. д.

Можно отметить следующие факторы, которые приводят к трудностям при обнаружении и распознавании объектов:

- непредсказуемость характера исходного изображения;

- нерегулярное движение фона;

- шумовые и регулярные изменения яркости изображения, как по всему полю, так и в отдельных пикселях, затрудняющих идентификацию одного и того же объекта в разных кадрах;

- шумы съемки, передачи информации и оцифровка, сопоставимые по тоновым представлениям с характеристиками распознаваемых объектов;

- сложный природный фон и возможность частичного перекрытия между собой объектов, а также объекта и элемента фона.

Эффективность поиска объектов наблюдения зависит от яркости объекта; контраста объект/фон; угловых размеров объекта; угловых размеров поля обзора; времени наблюдения объекта; скорости движения объекта [2]. 
Компьютерный анализ изображений относится к таким задачам обработки данных, в которых объем вычислений прямо пропорционален квадрату размерности изображений и числу рассматриваемых спектральных каналов этого изображения [3].

В связи с этим, для обеспечения необходимой вычислительной мощности, раньше обработка изображений была ориентирована на применение больших ЭВМ или мини-ЭВМ со специализированными системами обработки. Однако в настоящее время в связи с мощностью вычислительных машин, появились перспективы разработки новых математических методов и алгоритмов обработки изображений с использованием их в прикладных автоматизированных системах. Сочетание отмеченных факторов позволяет создавать системы обработки изображений, которые можно реализовать с помощью вычислительных машин и которые будут обладать удовлетворительными временными характеристиками [4].

Можно рассматривать процесс распознавания как последовательное преобразование графических данных от одного уровня представления к другому, что имеет высокий уровень обобщения информации в исходное изображение. Конечная цель обработки в данном случае заключается в обобщении данных полученных с устройства ввода, до уровня, необходимого для завершающей количественной обработки и получения необходимых характеристик [3 - 7].

Операция предобработки применяется практически всегда после снятия информации с видео датчика. Предобработка имеет целью снижение помех изображения, которые возникли в результате дискретизации и квантования, а также подавления внешних шумов. Как правило, это операции усреднения и выравнивания гистограмм.

Алгоритм удаления шума состоит из четырех этапов:

- сортировка пикселей в порядке возрастания их яркости;

- удаление «белого» шума;

- сортировка пикселей в порядке убывания их яркости;

- удаление «черного» шума.

Обработка пикселей выполняется в течение двукратного сканирования с использованием дополнительного массива длиной равной количеству уровней яркости [8] .

Удаление «белого» шума осуществляется при первом сканировании упорядоченных точек. Пусть $t_{i}$ будет пикселем с яркостью $v\left(t_{i}\right)$. Обработка пикселя состоит из проверки на содержание «белого» шума в области $G$, которая содержит этот пиксель. Сначала область содержит только $t_{i}$. Затем область растет так, что некоторые пиксели $t^{\prime}$ входят в нее, если:

- $t^{\prime}$ соседствует области $G$;

$-\quad v\left(t^{\prime}\right) \geq v\left(t_{i}\right)$.

Регион растет, пока не исполнится одно из следующих условий:

- размер полученной области $G$ превышает определенный размер. В этом случае область $G$ не рассматривается как шум и обрабатывается следующий $t_{i+1}$ пиксель;

- пиксель не может быть включен в $G$. В этом случае $G$ является шумом и яркость всех его точек составляют максимальную яркость соседних пикселей.

Удаление «черного» шума похоже на удаление «белого» шума. 
Для описания и сегментации свойств изображений, именно, однородности, шероховатости, регулярности, применяют методы текстур делящихся условно на две категории: статистические и структурные. Примером статистического подхода является использование матриц совпадений, формируемых из исходных изображений, с последующим подсчетом статистических моментов и энтропии. При структурном подходе, например, на основе мозаики Вороного, строится множество многоугольников. Многоугольники с общими свойствами объединяют в области. Для исследования общих свойств часто используются такие признаки как моменты многоугольников.

После сегментации возникают помехи в виде, как разрозненных изменений изолированных элементов изображения, так и в виде искажений некоторых связных областей [9].

Распознавание формулируется как частный случай классической задачи «наилучшего совпадения». Пусть $\ell$ будет изображением некоторого идеального объекта. Изображение зависит от его геометрических параметров: масштаба $\mu$, смещения $\xi$ и $\eta$, а также вращения $\varphi$, так что $\ell=\ell(\xi, \eta, \mu, \varphi)$. Пусть $\rho$ будет анализируемым изображением. Пусть $R(\rho, \ell)$ будет функцией непохожести, которая показывает, насколько реальная картина $\rho$ подобна идеальной картине $\ell$. Задача состоит в нахождении таких смещений $\xi^{*}$ и $\eta^{*}$, масштаба $\mu^{*}$ и поворота $\varphi^{*}$ от идеальной картины, которые обеспечивают лучшее сходство реальной картины к идеальной картине. Задача сводится к вычислению (1):

$$
\left(\xi^{*}, \eta^{*}, \mu^{*}, \varphi^{*}\right)=\arg \min R(\rho, \ell(\xi, \eta, \mu, \varphi))
$$

Хорошо известно, что порог бинаризации изображения можно предварительно определить так, что одно и то же значение будет хорошо подходить к любому изображению. В работе [10] порог зависит от обеих фотографий, сходство которых необходимо вычислить.

В настоящей статье сформированы и описаны результаты исследований задачи обнаружения объекта наблюдения на изображении, сформированы методы оценки несходства между фотографиями и исследованы вопросы предварительной обработки изображения.

\section{Исследование проведено под руководством:}

- доктора технических наук, профессора, Кузьмук Валерия Валентиновича, академика Международной академии информатизации, заместителя руководителя Отделение гибридных моделирующих и управляющих систем в энергетике Института проблем моделирования в энергетике.

\section{References:}

1. Волошин Н.В. Моделирование и распознавание информативных участков в автоматизированных системах иридодиагностики / Н.В. Волошин, В.В. Кузьмук, Е.А. Тараненко - Харьков: Восточно-Европейский журнал передовых технологий. - 2011. - №2/2 (50) - c. 65-69.

2. Волошин М.В. Моделі опису об'єкта та достовірність ідентифікації в системах комп'ютерного зору. - Харьков: Восточно-Европейский журнал передовых технологий. - 2010. - №4/7 (46) - с. 56-63.

3. Дуда Р. Распознавание образов и анализ сцен / Р. Дуда, П. Харт - М.: Мир, 1976. $368 \mathrm{c}$. 
4. Писаревский А.Н. Системы технического зрения (принципиальные основы, аппаратное и математическое обеспечение) / А.Н. Писаревский, А.Ф. Чернявский, Г.К. Афанасьев - Машиностроение. Ленингр. отд-ние, 1988. - 424 с.

5. Абламейко С.В. Обработка изображений: технология, методы, применения. Учебное пособие / С.В. Абламейко, Д.М. Лагуновский - Минск.: Амалфея, 2000. $304 \mathrm{c}$.

6. Сойфер В.А. Методы компьютерной обработки изображений. - М.: Физматлит, 2001. - 784 c.

7. Линдли К. Практическая обработка изображений на языке Си: Пер. с англ. - М.: Мир, 1996. - 512 с.

8. He Z. Toward Accurate and Fast Iris Segmentation for Iris Biometrics / Z. He, T. Tan, Z. Sun, X. Qiu - Transactions on Pattern Analysis and Machine Intelligence - Vol. 31 2009.

9. Viola P. Robust Real-Time Face Detection / P. Viola, M. Jones - International Journal of Computer Vision. - 2004. - №57(2). - pp. 137-154.

10. Aksak I. Detection of the Objects With Given Shape on the Grey-Valued Pictures / I. Aksak, V. Matsello, M. Schlesinger and others - Proceedings of 7-th International Conference on CAIP. - Kiel, Germany: Springer, 1997. - pp. 551-558 